\title{
Neurofibroma invading into urinary bladder presenting with symptoms of obstructed defecation and a large perineal hernia
}

\author{
Duminda Subasinghe ${ }^{1}$, Chathuranga Tisara Keppetiyagama' ${ }^{1}$, Chandu De Silva², Neville D Perera ${ }^{3}$ \\ and Dharmabandhu N Samarasekera ${ }^{1 *}$
}

\begin{abstract}
Background: Pelvic floor hernias pose a diagnostic and a treatment challange. Neurofibromatosis is a rare systemic disease, and urinary tract involvement is rare.

Case presentation: Here we report a case of a 54-year-old female with multiple neurofibromatosis who presented with features of obstructed defecation and was found to have a large perineal hernia. At surgery, we found an unusual herniation of a large neuropathic bladder and rectum through a perineal defect. She underwent reduction cystoplasty and repair of the pelvic floor using a prolene mesh. Subsequent histopathological examination confirmed a large neurofibroma infiltrating the urinary bladder.

Conclusion: Neurofibromatosis of the bladder is rare it should be considered as a differential diagnosis in patients presenting with symptoms of obstructed defecation.
\end{abstract}

Keywords: Multiple neurofibromatosis, Obstructive defecation, Perineal hernia

\section{Background}

Perineal hernias are rare and to date, about 100 cases have been reported in the literature [1]. It represents the protrusion of intra peritoneal or extra peritoneal contents through a congenital or an acquired defect in the pelvic floor. The first case of an acquired perineal hernia was reported in 1939 by Yeomen [2] and that of a primary hernia was reported by De Garangeot in 1743 [3]. This rare condition could be a diagnostic and a management challenge. These conditions need to be differentiated from other causes of perineal pathologies such as lipoma, bartholian cyst etc. Acquired perineal hernias are primary or secondary. Primarily acquired perineal hernias are caused by factors associated with increased intra-abdominal pressure. They are more common in females as a result of the broader pelvis and the strectching of the pelvic floor during pregnancy and childbirth. Secondarily, acquired perineal hernias are incisional hernias associated

\footnotetext{
* Correspondence: samarasekera58@yahoo.co.uk

'The National Hospital of Sri Lanka, University Surgical Unit, Colombo, Sri Lanka

Full list of author information is available at the end of the article
}

with extensive pelvic operations such as abdominoperineal resection, pelvic exenteration and perineal prostatectomy. Here we describe a patient with type 1 multiple neurofibromatosis who presented with features of obstructed defecation and a perineal hernia. She underwent surgery and was found to have the urinary bladder infiltrated with neurofibroma on subsequent histopathology.

\section{Case presentation}

A 54 year old female presented with a history of a swelling in her right buttock of 6 years duration. Her main complaint was difficulty in defecation when sitting due to a large lump in the perineum (Figure 1). She also had features of obstructed defecation syndrome such as excessive straining with unsuccessful attempts to evacuate, prolonged episodes in the toilet, rectal pain, rectal digitation and laxative dependency. Obstructed defecation symptoms were not relieved by regular use of laxatives. She had no obstructive urinary symptoms. She had not undergone any major gynecological procedures.

She was a diagnosed patient with multiple neurofibromatosis type 1 since the age of 17 years and had 


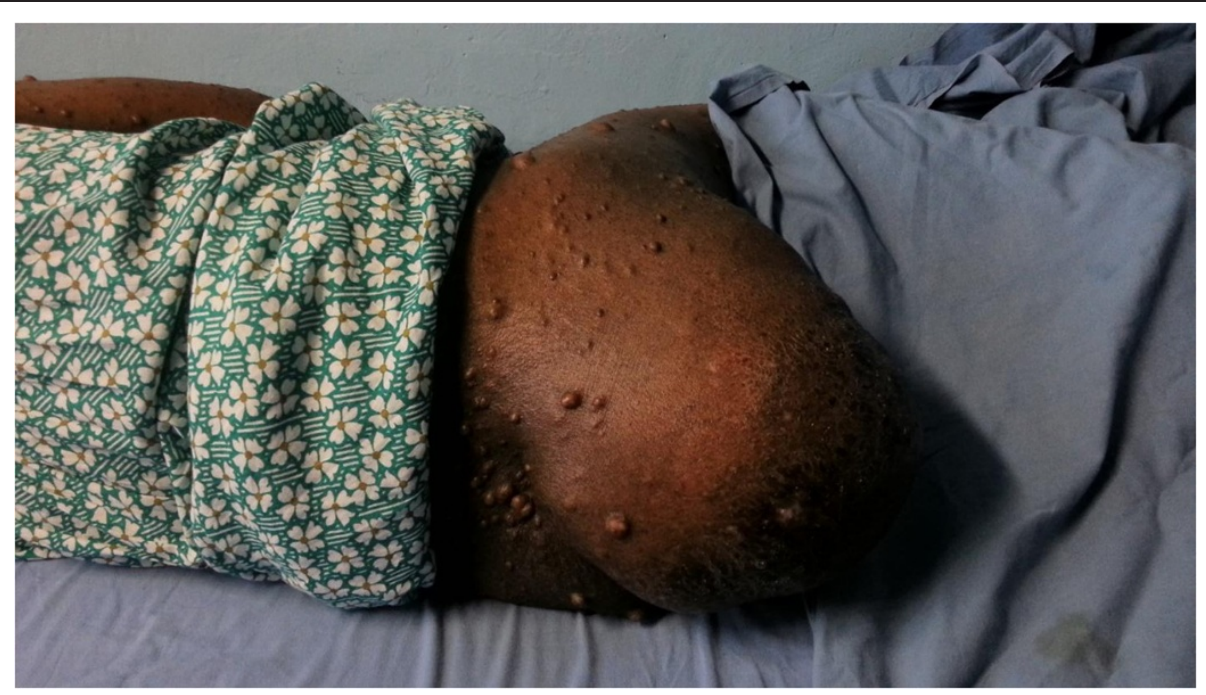

Figure 1 Large R/sided buttock hernia.

undergone excision of neurofibromas adjacent to right knee joint and over the right buttock 7 years back. She did not have a family history of neurofibromatosis. She did not give a past history of hypertension or other comorbidities. On examination, she had multiple neurofibromas all over the body and one plexiform neurofibroma over left knee area. There was a non reducible lump of $25 \times 20 \mathrm{~cm}$ size over the right buttock. It had an expansile cough impulse and was non tender on palpation. Digital rectal examination was normal. Therefore, a clinical diagnosis of a secondary perineal hernia was made.

Contrast enhanced computed tomography showed a large poorly enhancing mass in the region of right ischiorectal fossa suggestive of herniation of a large neuropathic bladder (Figure 2) and rectum through right ischiorectal

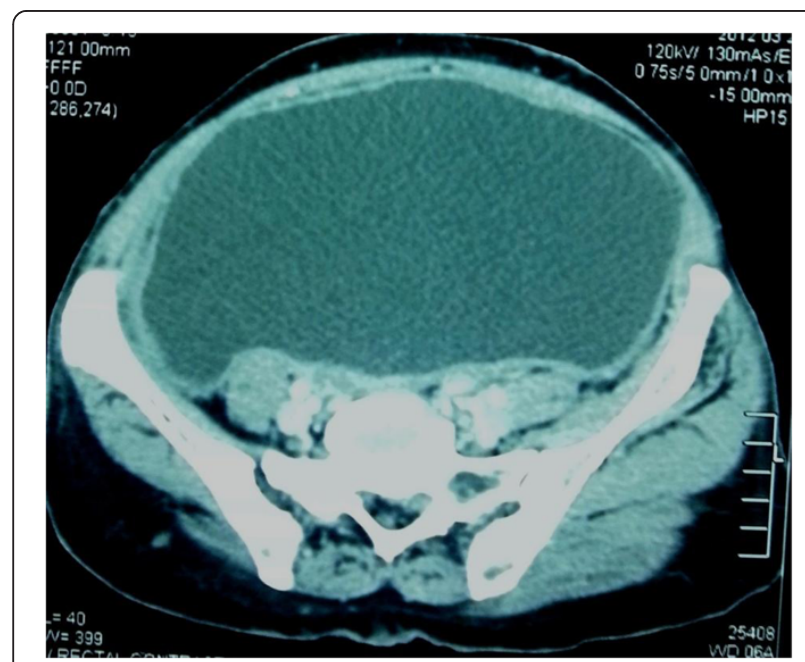

Figure 2 CT pelvis showing large neuropathic urinary bladder.

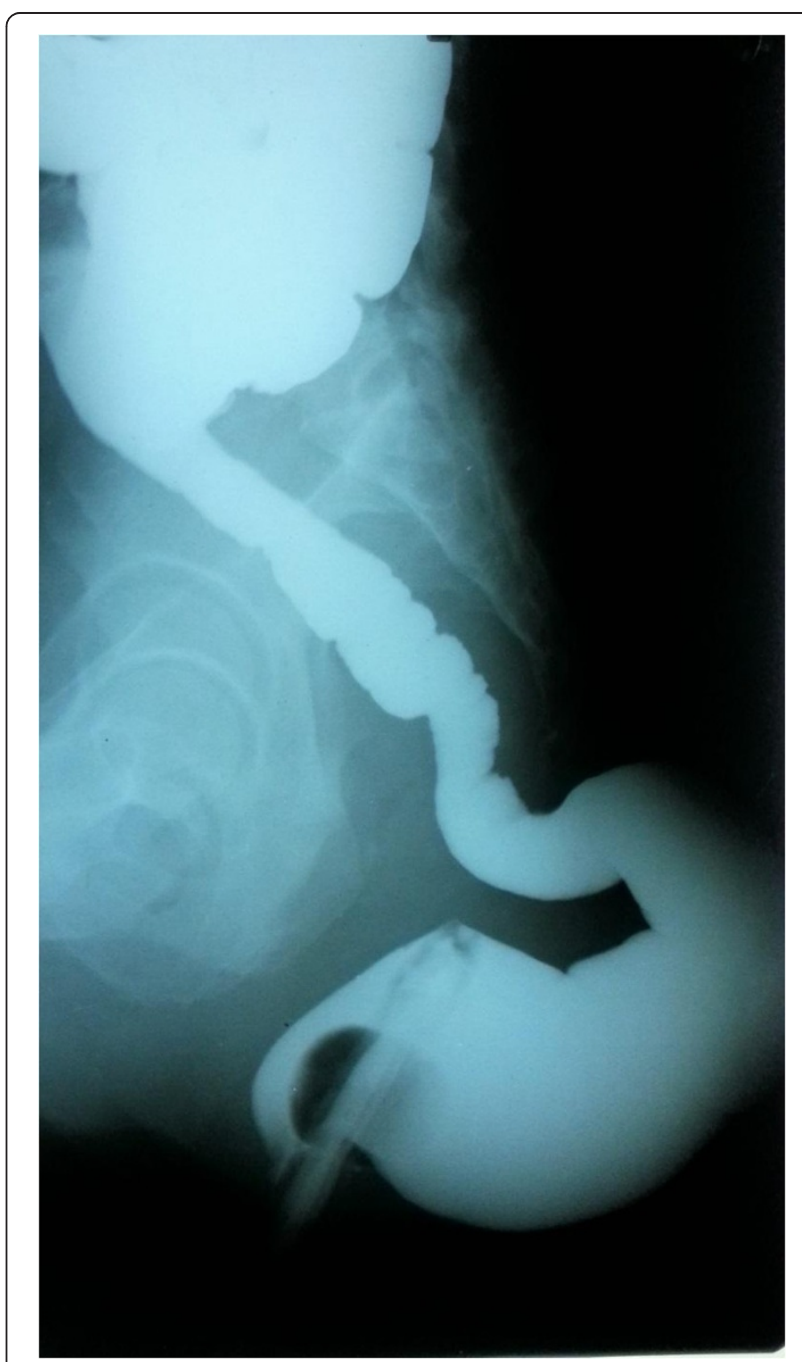

Figure 3 Barium enema showing the herniated rectum. 


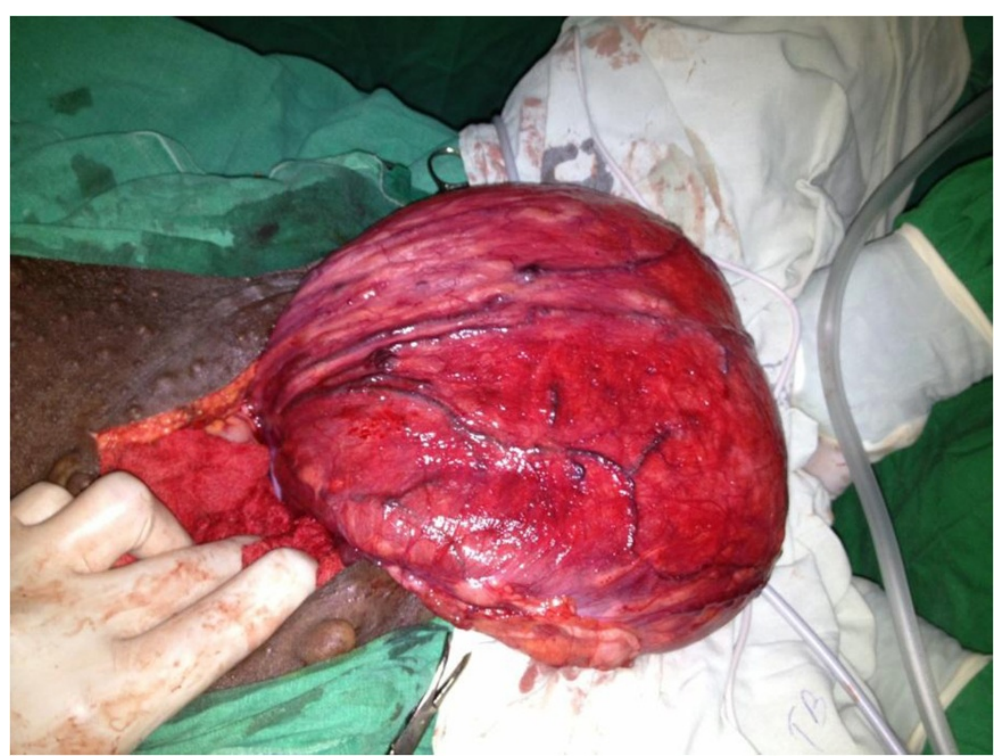

Figure 4 Large neuropathic bladder found during surgery.

fossa. Her US scan of the abdomen revealed mild right sided hydronephrosis. She underwent sigmoidoscopy which was normal up to the proximal sigmoid colon. Barium enema (Figure 3) showed herniation of the rectum through a defect in the pelvic floor.

The patient was planned for a hernia repair by a combined surgery with participation of colorectal, urological and plastic surgical teams. The patient was positioned on Lloyd Davies position and the repair of her hernia was done using an abdominal approach with a lower mid line incision. There was a large hernia sac extending in to right buttock area which contained a large neuropathic urinary bladder (Figure 4). A defect was noticed in the levator ani posteriorly on the right side, through which the urinary bladder and a portion of rectum had herniated. Openings of the ureters were not demonstrated and the right ureter was traced up to pelvic brim and stenting done. Cystectomy and reduction cystoplasty was followed by suprapubic catheterization (Figure 5). Hernial orifice was repaired with a prolene mesh. She was discharged six weeks following surgery. Currently she is on regular clinic follow up and intermittent urethral catheterization training.

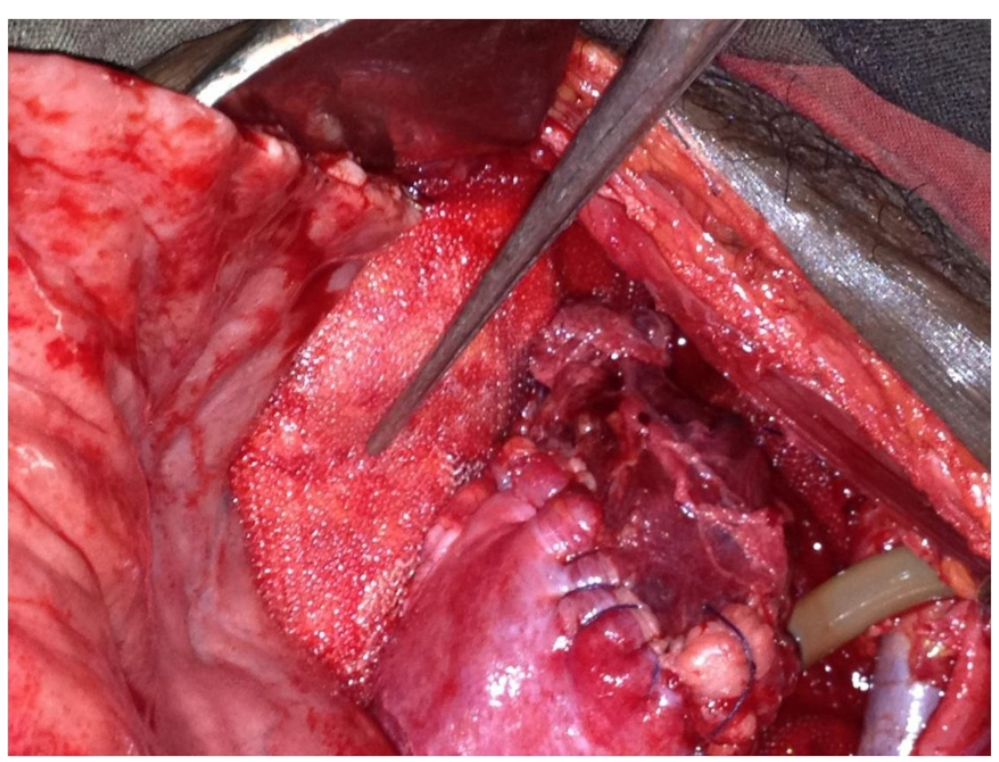

Figure 5 Repaired bladder with supra pubic catheter and mesh placed in the pelvic floor defect. 


\section{Discussion}

NF-1 is an autosomal dominant disorder characterized by multiple neurofibromas, multiple cutaneous café au lait spots, axillary freckling, optic nerve gliomas and skeletal abnormalities. It is one of the more common genetic disorders with a frequency of 1 in 3000 live births [4-6]. It is a multisystem disorder that may affect any organ in the body [7]. Visceral involvement in disseminated NF is rare. Involvement of the urinary tract by neurofibromatosis is uncommon. Neurofibromas of the genito-urinary tract have been shown to derive from nerves of the pelvic, vesicle and prostatic plexuses. The bladder is the commonest affected organ in the urinary tract, either as an isolated mass or a diffuse infiltrative process as in the cases described in this report. In 1878 Gerhardt [8] first described urinary tract involvement with a case of neurofibromatosis in the bladder and spinal cord and more than a century later a total of 75 cases have been reported of which less than a third are paediatric [9]. So far only four cases of malignant transformation of neurofibromatosis of the bladder have been reported [10-12]. Urinary bladder neurofibroma may present with LUTS, flank pain or enuresis and incontinence. But our patient presented with a large buttock mass and features of obstructed defecation syndrome.

Imaging plays an important role in the diagnosis, evaluation and follow-up of patients with abdominal manifestations of NF1. Barium studies may demonstrate intraluminal mass lesions. Our patient's barrium enema was suggestive of a displaced rectum and sigmoid colon with herniation of rectum through the pelvic floor. Anatomically, perineal hernias are classified as anterior or posterior, depending on the relationship to the transverse perineal muscle. The posterior is the less common variety and occurs through a defect in the levator ani muscle or between the levator ani and the coccygeus muscle $[13,14]$. Our patient had a posterior perineal hernia where there was a defect in the levator ani muscle. The techniques of repair through a transabdominal, a perineal, or combined transabdominal and perineal approaches have been described. Principles of surgical repair includes reduction of the sac contents and repair of the pelvic floor defect with either direct suture or implantation of a mesh [13].

\section{Conclusions}

Eventhough neurofibromatosis of the bladder is rare it should be considered as a differential diagnosis in patients presenting with symptoms of obstructed defecation specially with evidence of von Recklinghausen's disease. According to the authors' knowledge, this is the first report of neurofibromatosis involving the urinary bladder presenting as a buttock hernia causing obstructed defecation.

\section{Consent}

Written informed consent was obtained from the patient for publication of this case report and any accompanying images. A copy of the written consent is available for review by the Editor-in-Chief of this journal.

\section{Competing interests \\ The authors declare that they have no competing interests.}

\section{Authors' contribution}

All authors contributed to management of the patient and contributed equally to drafting of the manuscript. DNS and DS provided overall supervision and edited the final version of the manuscript. All authors have read and approved the final manuscript.

\section{Acknowledgement}

The authors acknowledge all the ward staff who took care of the patient.

\section{Author details}

'The National Hospital of Sri Lanka, University Surgical Unit, Colombo, Sri Lanka. ${ }^{2}$ Department of Pathology, University of Colombo, Colombo, Sri Lanka. ${ }^{3}$ Department of Urology and renal transplant, The National Hospital of Sri Lanka, Colombo, Sri Lanka.

Received: 13 November 2013 Accepted: 8 April 2014

Published: 16 April 2014

\section{References}

1. Preiss A, Herbig B, Do" rner A: Primary perineal hernia: a case report and review of the literature. Hernia 2006, 10(5):430-433.

2. Yeoman FC: Levator hernia perineal and pudendal. Am J Surg 1939, 43:695-697.

3. Stamatiou D, Skandalakis JE, Skandalakis LJ, Mirilas P: Perineal Hernia: surgical anatomy, embryology, and technique of repair. Am Surg 2010, 76(5):474-479.

4. Weiss SW, Goldblum JR: Benign tumors of peripheral nerves. In Enzinger and Weiss's soft tissue tumors. 4th edition. Edited by Strauss M. St Louis, Missouri: Mosby; 2001:1126-1146.

5. Riccardi VM, Eichner JE: Neurofibromatosis:Phenotype natural history and pathogenesis. Baltimore: Johns Hopkins University Press; 1986.

6. Ishii S, Han S, Shiiba K, Mizoi T, Okabe M, Horii A, Nagura H, Matsuno S, Sasaki l: Allelic loss of the NF1 gene in anal malignant melanoma in a patient with neurofibromatosis type 1. Int J Clin Oncol 2001, 6(4):201-204.

7. Rastogi R: Intra-abdominal manifestation of vonRecklinghausen's neurofibromatosis. Saudi J Gastroenterol 2008, 14:80-82.

8. Gerhardt C: Zur diagnostik multipler neurobildung. Dtsch Arch Klin Med 1878, 21:268.

9. Hintsa A, Lindell O, Heikkila P: Neurofibromatosis of the bladder, case report. Scand J Urol Nephrol 1996, 30:497-499.

10. Hulse CA: Neurofibromatosis: bladder involvement with malignant degeneration. J Urol 1990, 144:742-743.

11. Rober PE, Smith JB, Sakr W, Pierce JM: Malignant peripheral nerve sheath tumour (malignant schwannoma) of urinary bladder in von Recklinghausen neurofi-bromatosis. Urology 1991, 38:473-476.

12. Dahm P, Manseck A, Flossel C, Saeger HD, Wirth M: Malignant neurofibroma of the urinary bladder. Eur J Urol 1995, 27:261-263.

13. Salameh JR: Primary and unusual abdominal wall hernias. Surg Clin N Am 2008, 88(1):45-60.

14. Lubat E, Gordon RB, Birnbaum BA, Megibow AJ: CT diagnosis of posterior perineal hernia. AJR Am J Roentgenol 1990, 154(4):761-762.

doi:10.1186/1471-2482-14-21

Cite this article as: Subasinghe et al: Neurofibroma invading into urinary bladder presenting with symptoms of obstructed defecation and a large perineal hernia. BMC Surgery 2014 14:21. 